Pacific Journal of Mathematics

TESTING 3-MANIFOLDS FOR PROJECTIVE PLANES 


\title{
TESTING 3-MANIFOLDS FOR PROJECTIVE PLANES
}

\section{WOLFGANG HEIL}

\begin{abstract}
It is well known that a closed 3-manifold $M$ contains a (piecewise linearly embedded) essential separating 2-sphere if and only if $\pi_{1}(M)$ is a nontrivial free product. In this paper necessary and sufficient conditions, in terms of $\pi_{1}(M)$, are given for the existence of a projective plane in $M$. If $M$ is irreducible this condition is that $\pi_{1}(M)$ be an extension of $Z$ or a nontrivial free product by $Z_{2}$. In particular this provides a criterion for deciding which irreducible closed 3 -manifolds are not $P^{2}$-irreducible.
\end{abstract}

$P^{2}$-irreducible 3-manifolds have been studied in [2], [4]; if they are sufficiently large then their covering spaces are also $P^{2}$-irreducible. This property is not shared by irreducible but not $P^{2}$-irreducible manifolds; in [9] such manifolds are constructed having non prime covering spaces. This leads to the question as to which 3-manifolds are irreducible but not $P^{2}$-irreducible.

o. Notation and definitions. We work in the piecewise linear category. A 3-manifold $M$ is a compact, connected 3-manifold. $A$ surface $F$ in $M$ is a compact 2-manifold embedded in $M$.

We denote by $U(X)$ a small regular neighborhood of $X$ in $M$.

$F \subset \operatorname{Int}(M)$ is 2-sided in $M$ if $U(F)$ is homeomorphic to $F \times I$. $M$ is irreducible if every 2 -sphere in $M$ bounds a 3 -cell in $M . M$ is $P^{2}$-irreducible if $M$ is irreducible and contains no 2-sided projective planes. $M$ is prime if it is not the connected sum of two manifolds each different from the 3-sphere. (Here the connected sum $M_{1} \# M_{2}$ is obtained by removing a 3-ball in the interior of $M_{1}$ and $M_{2}$ and identifying the boundary spheres under an orientation reversing homeomorphism.) $F$ in $M$ is incompressible if the following holds:

(a) if $D$ is a disc in $M$ such that $D \cap F=\partial D$, then $\partial D$ bounds a dise in $F$, and

(b) if $F$ is a 2-sphere, then $S$ does not bound a 3-ball in $M$.

A homotopy $N$ is a manifold that is homotopy equivalent to the manifold $N$.

Disjoint surfaces $F$ and $G$ in $M$ are pseudo parallel if there exists an embedding of a homotopy $(F \times I)$ into $M$ that has two boundary components, one of which is mapped onto $F$, the other one onto $G$. Finally, $M$ is called $\pi$-trivial, if $\pi_{1}(M)=1$.

REMARK. If the Poincare conjecture is true, then pseudo parallel 
is the same as parallel.

1. Preliminaries. Let $S^{2}, P^{2}$ denote the 2-sphere and projective plane, resp.

LEMMA 1. Let $F$ be a closed surface, let $M$ be an irreducible 3manifold.

(a) If $F \neq S^{2}, P^{2}$ then $M$ is a homotopy $(F \times I)$ if and only if $M$ is homeomorphic to a line bundle over $F$.

(b) If $M$ is nonorientable and $\pi_{1}(M)=Z_{2}$, then $\partial M$ consists of two projective planes and $M$ is a homotopy $\left(P^{2} \times I\right)$.

(c) If $\pi_{1}(M)=\boldsymbol{Z}+\boldsymbol{Z}_{2}$, then $\partial M=\varnothing$ and $M$ is a homotopy $\left(P^{2} \times S^{1}\right)$.

Proof. Part (a) follows from [5, Proposition 1]. Part (b) follows from [1, Theorem 5.1]. Part (c) follows from [11]: We map $M$ onto a circle such that the inverse image of a point is a projective plane $P^{2}$ in $M$. Then, by (b), cl $\left(M-U\left(P^{2}\right)\right)$ has as boundary two copies of $P^{2}$ and is a homotopy $\left(P^{2} \times I\right)$.

LEMMA 2. If $M$ is irreducible and contains a 1-sided projective plane, then $M$ is $P^{3}$ (the 3-dim. projective space).

Proof. $U\left(P^{2}\right)$ is the twisted line bundle over $P^{2}$, with boundary a 2-sphere. Since this 2-sphere bounds a 3-cell in $M$, the result follows.

The next lemma is due to J. Tollefson [13, Lemma 1]:

Lemma 3. A non-irreducible closed 3-manifold $M$ admitting a fixed point free involution $T$ contains a 2-sphere $S$ not bounding a 3-cell in $M$ such that either $T(S)=S$ or $T(S) \cap S=\varnothing$.

We will also need the following generalization of Tollefson's lemma.

LEMMA 4. Let $M$ be a 3-manifold (with or without boundary) admitting a fixed point free involution $T$. Suppose there exists a 2sphere in $M$ that does not separate $M$ into two components one of which is $\pi$-trivial. Then there exists a 2-sphere $S$ in $M$ having the same property and such that either $T(S) \cap S=\varnothing$ or $T(S)=S$.

Proof. Take a 2-sphere $S$ in $M$ with the following properties: $S$ does not separate $M$ into two components one of which is $\pi$-trivial, $T(S) \cap S$ is a system of disjoint simple closed curves at which the intersection is transversal, and the number $n(T(S) \cap S)$ of components $T(S) \cap S$ is minimal. We show that either $n=0$ or there exists an $S^{\prime}$ with 
the desired properties such that $T\left(S^{\prime}\right)=S^{\prime}$.

Suppose $n>0$. Let $D$ be an innermost disc on $T(S)$, with $\partial D$ a component of $T(S) \cap S$, (that is, int $(D) \cap S=\varnothing$ ). $D$ separates $S$ into two discs $D_{1}, D_{2}$. Let $S_{1}=D \cup D_{1}, S_{2}=D \cup D_{2}$. It is easy to see that at least one of $S_{1}$ or $S_{2}$ does not separate $M$ into two components one of which is $\pi$-trivial. Suppose $S_{1}$ has this property. If $T\left(S_{1}\right)=S_{1}$, we are done. If $T\left(S_{1}\right) \neq S_{1}$, then a component $S^{\prime}$ of $\partial U\left(S_{1}\right)$ ( $U$ is small wrt $T$ ) has the same property as $S_{1}$, but $n\left(T\left(S^{\prime}\right) \cap S^{\prime}\right)<$ $n(T(S) \cap S)$ (since the component $\partial D$ has vanished), a contradiction.

Lemma 5. If $M$ is closed and $\pi_{1}(M) \approx Z$, then $M$ is a connected sum of a homotopy 3-sphere and a $S^{2}$-bundle over $S^{1}$.

Proof. Write $M \approx M_{1} \# M_{2}$, where $M_{1}$ is prime and $\pi_{1}\left(M_{1}\right) \approx Z$, $\pi_{1}\left(M_{2}\right)=1$ (see $\S 5$ ). An irreducible manifold with fundamental group $\boldsymbol{Z}$ is bounded (see e.g. [11]). Hence $M_{1}$ is not irreducible. Therefore $M_{1}$ is an $S^{2}$-bundle over $S^{1}$ (see $\S 5$ ).

\section{The closed case.}

Theorem 1. A closed irreducible 3-manifold $M$ contains a 2-sided projective plane if and only if $\pi_{1}(M)$ is an extension of $\boldsymbol{Z}$ or a nontrivial free product by $Z_{2}$.

Proof. Suppose $M$ contains a 2-sided $P^{2}$. Thus $M$ is nonorientable and we let $p: M^{\prime} \rightarrow M$ be the 2-fold orientable covering of $M$. Then $P^{2} \subset M$ lifts to an essential 2-sphere $S^{2} \subset M^{\prime}$. If $S^{2}$ separates $M^{\prime}$ into $M_{1}, M_{2}$ then $\pi_{1}\left(M^{\prime}\right) \cong \pi_{1}\left(M_{1}\right) * \pi_{1}\left(M_{2}\right)$, a nontrivial free product. (Otherwise, if $\pi_{1}\left(M_{1}\right)=1$, say, from $\partial M_{1}=S^{2}$ it would follow that $S^{2}$ is contractible in $M_{1}$ ). If $S^{2}$ does not separate $M^{\prime}$, let $k$ be a simple closed curve that intersects $S^{2}$ in exactly one point and let $U=$ $U\left(S^{2} \cup k\right)$. Then $\pi_{1}\left(M^{\prime}\right)=Z * \pi_{1}(\mathrm{cl}(M-U))$.

Conversely, assume $\pi_{1}(M)$ is an extension of $\boldsymbol{Z}$ or of a nontrivial free product $G$ by $Z_{2}$. Let $p: N \rightarrow M$ be the covering of $M$ associated with $Z$ or $G$, respectively, and let $T: N \rightarrow N$ be the covering transformation. By Lemma 5 and Kneser's conjecture [12] there exists an essential 2-sphere $S^{2}$ in $N$. Therefore, by Lemma 3 we can find a 2-sphere $S \subset N$ not bounding a 3-cell, such that either $T(S) \cap S=\varnothing$ or $T(S)=S$. The first case cannot occur, since $M$ is irreducible. In the second case, $p(S)$ is a projective plane in $M$ that is 2 -sided, by Lemma 2.

3. The bounded case. 
THeOREM 2. Let $M$ be an irreducible 3-manifold with (nonempty) incompressible boundary. $M$ contains a 2-sided $P^{2}$ that is not pseudo parallel to a component of $\partial M$ if and only if $\pi_{1}(M)$ is an extension of a nontrivial free product by $\boldsymbol{Z}_{2}$.

Proof. Suppose $M$ contains a 2-sided $P^{2}$ that is not pseudo parallel to a component of $\partial M$. Lift $P^{2}$ to $S^{2}$ in the 2 -fold orientable cover $M^{\prime}$ of $M$, let $T: M^{\prime} \rightarrow M^{\prime}$ be the covering transformation. If $S^{2}$ separates $M$ into $M_{1}, M_{2}$, we have that $T\left(M_{1}\right)=M_{1}, T\left(M_{2}\right)=M_{2}$, since $P^{2}$ is 2-sided in $M$. If $\pi_{1}\left(M_{1}\right)=1$, say, then $M_{1}$ covers a submanifold $M_{1_{*}}$ having fundamental group $\boldsymbol{Z}_{2}$. By Lemma 1 (b), $M_{1^{*}}$ is a homotopy $\left(P^{2} \times I\right)$, hence $P^{2}$ would be pseudo parallel to a component of $\partial M$, a contradiction. Therefore, in this case, $\pi_{1}\left(M^{\prime}\right)=\pi_{1}\left(M_{1}\right) * \pi_{1}\left(M_{2}\right)$, a nontrivial free product.

If $S^{2}$ does not separate $M^{\prime}$, then as in the proof of Theorem 1 , $\pi_{1}\left(M^{\prime}\right) \cong Z * \pi_{1}\left(\mathrm{cl}\left(M^{\prime}-U\right)\right)$. If $\pi_{1}\left(\mathrm{cl}\left(M^{\prime}-U\right)\right)$ would be trivial, then $\pi_{1}(M)=\boldsymbol{Z}+\boldsymbol{Z}_{2}$. By Lemma 1 (c), $M$ would be closed, a contradiction.

Conversely, suppose $\pi_{1}(M)$ is an extension of a nontrivial free product $G$ by $Z_{2}$. Again, let $N \stackrel{P}{\longrightarrow} M$ be the covering of $M$ corresponding to $G$ and let $T$ be the covering transformation. By Kneser's conjecture for bounded 3-manifolds [6] there exists a 2-sphere $S^{2}$ in $N$ that separates $N$ into $N_{1}, N_{2}$, both not $\pi$-trivial. By Lemma 4, there exists a 2-sphere $S$ that does not separate $N$ into two components one of which is $\pi$-trivial and such that $T(S)=S$ (the case $T S \cap S=$ $\phi$ cannot occur). By Lemma $2, S$ covers a 2 -sided $P^{2}$ in $M$. If $P^{2}$ were pseudo parallel to a component of $\partial M$, then lifting the corresponding homotopy $\left(P^{2} \times I\right)$ we see that $S$ would separate $N$ into two components, one of which would be $\pi$-trivial, a contradiction.

Proposition. Let $M$ be irreducible and suppose $\pi_{1}(M)$ is not $\boldsymbol{Z}_{2}$, and not an extension of $\boldsymbol{Z}$ or of a nontrivial free product by $\boldsymbol{Z}_{2}$. Then if $\partial M$ contains no $P^{2}$ (in particular, if $M$ is closed) it follows that $M$ contains no $P^{2}$.

Proof. If $M$ is orientable and contains a $P^{2}$, then $M=P^{3}$, by Lemma 2. If $M$ is nonorientable, let $M^{\prime}$ be the 2-fold orientable cover of $M$. If $\pi_{2}\left(M^{\prime}\right) \neq 0$, then the sphere theorem [14] gives us an essential 2-sphere in $M^{\prime}$ and as in the proof of the preceding theorems, we see that $\pi_{1}\left(M^{\prime}\right)=Z$ or a nontrivial free product. Therefore, $\pi_{2}\left(M^{\prime}\right)=0$ and hence $\pi_{2}(M)=0$. (In fact, $M$ is aspherical.) But any 2-sided $P^{2} \subset M$ would be essential [1, Lemma 6.3].

REMARK. A 2-sided $P^{2}$ in $M$ is incompressible in $M$. This follows 
from the loop theorem and Dehn's lemma [10]. In particular $\pi_{1}\left(P^{2}\right) \rightarrow$ $\pi_{1}(M)$ is an injection.

4. A counterexample to Theorem 2 if $M$ is not incompressible. Let $K$ be a solid Kleinbottle, $T$ a solid torus. Choose $n \geqq 1$ disjoint $\operatorname{discs} D_{1}, \cdots, D_{n}$ on $\partial K$ and a disc $D$ on $\partial T$. Let $M$ be the manifold obtained from $K$ by attaching $n$ copies of $T$ to $K$ at $D_{i}$ and $D$ ( $i=$ $1, \cdots, n)$. Then $M$ is irreducible and does not contain 2-sided projective planes (otherwise by the preceding remark, $\pi_{1}(M)$ would have an element of order 2 , but $\pi_{1}(M) \cong(n+1) Z$ ). However, the twofold orientable cover $M^{\prime}$ of $M$ has fundamental group $\pi_{1}\left(M^{\prime}\right) \cong(2 n+$ 1) $Z$, the free product of $2 n+1$ copies of $Z$, and therefore $\pi_{1}(M)$ is an extension of the nontrivial free product $(2 n+1) Z$ by $Z_{2}$.

5. The general case. Suppose $M$ is a compact 3-manifold such that $\partial M$ contains no 2-spheres. As in [8, Lemma 1] it follows that if $M$ is prime but not irreducible then $M$ is a $S^{2}$-bundle over $S^{1}$. If $M$ is not prime, then there exists a decomposition of $M$ into a finite number of prime manifolds

$$
M \approx M_{1} \# M_{2} \# \cdots \# M_{n},
$$

(if $M$ is nonorientable or with boundary see e.g. [3]). If $K$ denotes the nonorientable $S^{2}$-bundle over $S^{1}$ then since $K \# K \approx K \#\left(S^{2} \times S^{1}\right)$, we say that the decomposition (\#) is in normal form if at most one $M_{i} \approx K$. Then Milnor's proof in [8] can be generalized to yield the following:

Proposition. Any compact 3-manifold $M$ whose boundary contains no 2-spheres has a unique normal decomposition (\#) into prime manifolds. Each summand $M_{i}$ is irreducible or $S^{1} \times S^{2}$ and at most one $M_{i} \approx K$.

In the decomposition $(\#)$ let $m$ denote the number of prime mani. folds which are not $\pi$-trivial $(m \leqq n)$.

THeORem 3. Let $M$ be a closed 3-manifold.

(a) If $M$ contains a 2-sided $P^{2}$, then $\pi_{1}(M)$ is an extension of a free product of $2 m$ nontrivial factors or of a free product of $2 m-1$ nontrivial factors one of which is $\boldsymbol{Z}$, by $\boldsymbol{Z}_{2}$.

(b) If $\pi_{1}(M)$ is an extension of a free product of $2 m$ nontrivial factors by $Z_{2}$ then $M$ contains a 2-sided $P^{2}$.

Proof. Consider the decomposition (\#). Let $S_{i} \subset M$ be the 2sphere at which $M_{i}$ and $M_{i+1}$ are amalgamated and let $M_{i}^{\prime}$ be obtained 
from $M_{i}$ by removing the interiors of the 3-balls which are used in the construction of the connected sum. We can assume that $M_{i}^{\prime} \cap$ $M_{i+1}^{\prime}=S_{i}(i=1, \cdots, n-1)$.

We first note that $M$ contains a 2-sided $P^{2}$ if and only if one of the $M_{i}^{\prime}$ contains a 2 -sided $P^{2}$. For, by general position we can assume that $P^{2} \cap \cup S_{i}$ is a system of simple closed curves. If $P^{2} \cap S_{i} \neq \varnothing$ then an innermost intersection curve on $S_{i}$ bounds a disk on $P^{2}$ (since $P^{2}$ is incompressible) and on $S_{i}$. Replacing the disk on $P^{2}$ by the disk on $S_{i}$ and pushing it slightly off $S_{i}$, we reduce the number of intersection curves of $P^{2} \cap \cup S_{i}$.

Second, we note that we can assume that in the decomposition (\#) no $M_{i}$ has trivial fundamental group i.e. that $n=m$. For otherwise we consider the manifold $M_{*}$ obtained from $M$ by deleting all the homotopy spheres $M_{i}$ which occur in (\#). Clearly, $\pi_{1}\left(M_{*}\right)=\pi_{1}(M)$ and $M_{*}$ contains a 2-sided $P^{2}$ if and only if $M$ does.

Now assume $M$ contains a 2 -sided $P^{2}$. Let $p: N \rightarrow M$ be the 2fold orientable covering and let $N_{i}=p^{-1}\left(M_{i}^{\prime}\right)$. If $N_{i}$ is connected then $\pi_{1}\left(N_{i}\right) \neq 1$, because otherwise $\pi_{1}\left(M_{i}^{\prime}\right)=Z_{2}$, and since $\partial M_{i}^{\prime}$ consists of 2-spheres only, $M_{i}^{\prime}$ is orientable (Lemma $1(\mathrm{~b})$ ). But then $M_{i}^{\prime}$ lifts to two copies, hence $N_{i}$ would not be connected. Similarily, if $N_{i}$ is not connected then no component of $N_{i}$ is $\pi$-trivial, because otherwise $M_{i}$ would be $\pi$-trivial. Now each $S_{i} \subset M$ lifts to two 2-spheres $S_{i}^{\prime}, S_{i}^{\prime \prime}$ in $N$, and $N$ is obtained from the $N_{i}$ by identifying $N_{i}$ and $N_{i+1}$ along $S_{i}^{\prime}$ and $S_{i}^{\prime \prime}(i=1, \cdots, m-1)$.

Construct a manifold $N^{\prime}$ as follows. If both $N_{1}$ and $N_{2}$ are connected, identify $N_{1}$ and $N_{2}$ along one 2-sphere only, say $S_{1}^{\prime}$. Otherwise identify $N_{1}$ and $N_{2}$ along both $S_{1}^{\prime}$ and $S_{1}^{\prime \prime}$. The result is a manifold $N^{(1)}$. If $N_{3}$ is connected, identify $N^{(1)}$ and $N_{3}$ along $S_{2}^{\prime}$ only, otherwise identify along $S_{2}^{\prime}$ and $S_{2}^{\prime \prime}$, etc. In this way we obtain a maximal connected manifold $N^{\prime}$ such that $N$ is obtained from $N^{\prime}$ by identifying pairs of 2-spheres in $\partial N^{\prime}$. Then $\pi_{1}\left(N^{\prime}\right)=G_{1} * \cdots * G_{k}(0 \leqq k \leqq 2 m-1)$, where each $G_{j}$ is the fundamental group of a component of some $N_{i}$. We obtain $N$ from $N^{\prime}$ by adding $(2 m-1)-k$ handles $S^{1} \times S^{2}$ or $K$, hence $\pi_{1}(N)=G_{1} * \cdots * G_{k} * Z * \cdots * Z$ is a free product of $2 m-1$ nontrivial factors.

Now $P^{2} \subset M_{j}^{\prime}$, say $(1 \leqq j \leqq m-1)$. Then $M_{j}^{\prime}$ is nonorientable and $N_{j}$ is connected. Therefore by the above construction, $\pi_{1}\left(N_{j}\right)$, is one of the groups $G_{i}$ in the above decomposition of $\pi_{1}(N)$. Closing the boundary spheres of $N_{j}$ with 3 -balls we get a 2 -fold covering $\hat{N}_{j} \rightarrow M_{j}$, and it follows from the proof of Theorem 1 that $\pi_{1}\left(\hat{N}_{j}\right)$ and hence $\pi_{1}\left(N_{j}\right)$ is $Z$ or a nontrivial free product. This proves part (a) of Theorem 3 .

Now suppose $\pi_{1}(M)$ is an extension of a product $G$ of $2 m$ nontrivial groups by $Z_{2}$. Let $p: \widetilde{M} \rightarrow M$ be the covering associated to $G$. Then 
as above $\pi_{1}(\widetilde{M})=\pi_{1}\left(\widetilde{M}_{1}\right) * \cdots * \pi_{1}\left(\widetilde{M}_{k}\right) * Z * \cdots * \boldsymbol{Z}$ is a product of $2 m-1$ groups, where each $\tilde{M}_{i}$ is a component of $p^{-1}\left(M_{j}^{\prime}\right)$, for some $j$. (It is possible that some $\pi_{1}\left(\widetilde{M}_{i}\right)=1$.) It follows from Kurosh's Theorem [7] that at least one factor, $\pi_{1}\left(\widetilde{M}_{1}\right)$ say, is a nontrivial free product. If $\widetilde{M}_{1}$ covers $M_{j}^{\prime}$, then either $\pi_{1}\left(M_{j}\right) \approx \pi_{1}\left(\widetilde{M}_{1}\right)$ or $\pi_{1}\left(M_{j}\right)$ is an extension of $\pi_{1}\left(\tilde{M}_{1}\right)$ by $Z_{2}$. In the first case $M_{1}$ can not be a handle and by Kneser's conjecture can not be irreducible, therefore this case can not occur. In the second case we apply Theorem 1 to obtain a $P^{2}$ in $M_{1}$ and hence in $M$.

It should be noted that the hypothesis in case (a) of Theorem 3 can not be weakened: If $M=\left(P^{2} \times S^{1}\right) \#\left(S^{2} \times S^{1}\right)$, then $\pi_{1}(M)$ is not an extension of a free product of 4 factors by $\boldsymbol{Z}_{2}$.

It is now easy to see how to obtain an analogous result for 3manifolds with incompressible boundary.

\section{REFERENCES}

1. D. B. A. Epstein, Projective planes in 3-manifolds, Proc. London Math. Soc., (3) 11 (1961), 469-84.

2. C. D. Feustel, A generalization of Kneser's conjecture, to appear.

3. W. Haken, Ein Verfahren zur Aufspaltung einer 3-Mannigfaltigkeit in irreduzible 3-Mannigfaltigkeiten, Math. Z., 76 (1961), 427-467.

4. W. H. Heil, On P2-irreducible 3-manifolds, Bull. Amer. Math. Soc., 75 (1969), $772-$ 775 .

5. - On the existence of incompressible surfaces in certain 3-manifolds II, Proc. Amer. Math. Soc., 25 (1970), 429-432.

6. On Kneser's conjecture for bounded 3-manifolds, Proc. Cambridge Phil. Soc., 71 (1972), 243-246.

7. A. G. Kurosh, The theory of groups II, Chelsea, N. Y. 1960.

8. J. Milnor, A unique decomposition theorem for 3-manifolds, Amer. J. Math., 84 (1962), 1-7.

9. W. H. Row, Jr., Irreducible 3-manifolds whose orientable coverings are not prime, Proc. Amer. Math., Soc., 34 (1972), 541-545.

10. J. Stallings, On the loop theorem, Annals of Math., 72 (1960), 12-19.

11. On Fibering Certain 3-manifolds, Top. of 3-manifolds, (1962) Prentice Hall, Englewood, N. J.

12. - Grushko's Theorem II, Kneser's conjecture, Notices Amer. Math. Soc., 6 (1959), Abstract 559-165, 531-532.

13. J. Tollefson, Free involutions on non-prime 3-manifolds, Osaka J. Math., 7 (1970), 161-164.

14. J. H. C. Whitehead, 2-spheres in 3-manifolds, Bull. Amer. Math. Soc., 64 (1958), 161-166.

Received August 11, 1971 and in revised form April 7, 1972. Partially supported by NFS grant GP 19964.

The Florida State University 



\section{PACIFIC JOURNAL OF MATHEMATICS}

\section{EDITORS}

H. SAMELSON

Stanford University

Stanford, California 94305

C. R. Hовву

University of Washington

Seattle, Washington 98105
J. DugundJI

Department of Mathematics University of Southern California

Los Angeles, California 90007

RICHARD ARENS

University of California

Los Angeles, California 90024

\section{ASSOCIATE EDITORS}

E. F. BECKENBACH

B. H. NeumanN

F. WOLF

K. YoSHIDA

\section{SUPPORTING INSTITUTIONS}

UNIVERSITY OF BRITISH COLUMBIA

CALIFORNIA INSTITUTE OF TECHNOLOGY

UNIVERSITY OF CALIFORNIA

MONTANA STATE UNIVERSITY

UNIVERSITY OF NEVADA

NEW MEXICO STATE UNIVERSITY

OREGON STATE UNIVERSITY

UNIVERSITY OF OREGON

OSAKA UNIVERSITY
UNIVERSITY OF SOUTHERN CALIFORNIA

STANFORD UNIVERSITY

UNIVERSITY OF TOKYO

UNIVERSITY OF UTAH

WASHINGTON STATE UNIVERSITY

UNIVERSITY OF WASHINGTON

AMERICAN MATHEMATICAL SOCIETY

NAVAL WEAPONS CENTER

Printed in Japan by International Academic Printing Co., Ltd., Tokyo, Japan 


\section{Pacific Journal of Mathematics}

\section{Vol. 44, No. $1 \quad$ May, 1973}

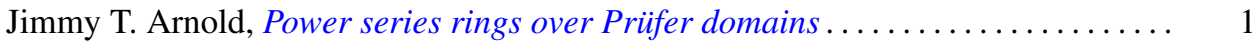

Maynard G. Arsove, On the behavior of Pincherle basis functions . . . . . . . . . 13

Jan William Auer, Fiber integration in smooth bundles ................. 33

George Bachman, Edward Beckenstein and Lawrence Narici, Function algebras

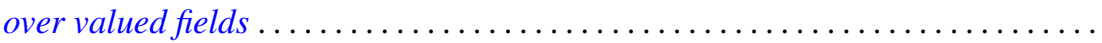

Gerald A. Beer, The index of convexity and the visibility function . . . . . . . . . . .

James Robert Boone, A note on mesocompact and sequentially mesocompact

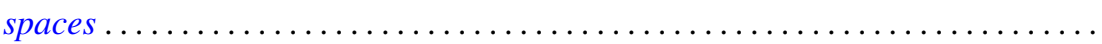

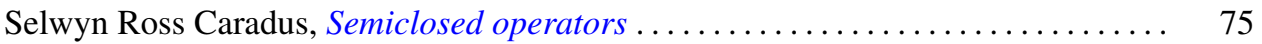

John H. E. Cohn, Two primary factor inequalities . . . . . . . . . . . . . . . 81

Mani Gagrat and Somashekhar Amrith Naimpally, Proximity approach to

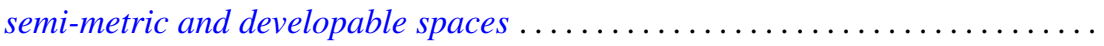

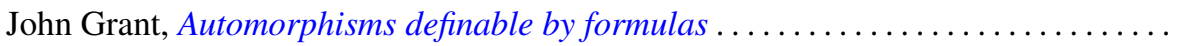

Walter Kurt Hayman, Differential inequalities and local valency ..............

Wolfgang H. Heil, Testing 3-manifolds for projective planes . . . . . . . . . . . . .

107

Melvin Hochster and Louis Jackson Ratliff, Jr., Five theorems on Macaulay

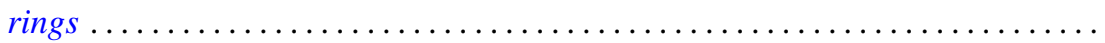

Thomas Benton Hoover, Operator algebras with reducing invariant subspaces ....

James Edgar Keesling, Topological groups whose underlying spaces are separable

Fréchet manifolds...

Frank Leroy Knowles, Idempotents in the boundary of a Lie group . .

191

George Edward Lang, The evaluation map and EHP sequences ...

201

Everette Lee May, Jr, Localizing the spectrum . . . . . . . . . . . .

211

Frank Belsley Miles, Existence of special $K$-sets in certain locally compact abelian groups.

Susan Montgomery, A generalization of a theorem of Jacobson. II . .

T. S. Motzkin and J. L. Walsh, Equilibrium of inverse-distance forces in

three-dimensions.

Arunava Mukherjea and Nicolas A. Tserpes, Invariant measures and the converse

of Haar's theorem on semitopological semigroups .

James Waring Noonan, On close-to-convex functions of order $\beta$

Donald Steven Passman, The Jacobian of a growth transformation

Dean Blackburn Priest, A mean Stieltjes type integral ........ .

Joe Bill Rhodes, Decomposition of semilattices with applications to topological

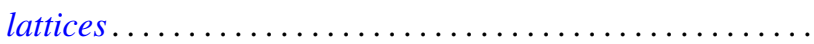

Claus M. Ringel, Socle conditions for $\mathrm{QF}-1$ rings ..........

Richard Rochberg, Linear maps of the disk algebra

Roy W. Ryden, Groups of arithmetic functions under Dirichlet convolution . .

Michael J. Sharpe, A class of operators on excessive functions

Erling Stormer, Automorphisms and equivalence in von Neumann algebras ..

Philip C. Tonne, Matrix representations for linear transformations on series

analytic in the unit disc. 